\title{
A mouse model of juvenile hemochromatosis
}

\author{
Franklin W. Huang, ${ }^{1}$ Jack L. Pinkus, ${ }^{2}$ Geraldine S. Pinkus, ${ }^{2}$ Mark D. Fleming, ${ }^{1}$ \\ and Nancy C. Andrews ${ }^{1,3}$ \\ ${ }^{1}$ Children's Hospital Boston, Dana-Farber Cancer Institute, Harvard Medical School, Boston, Massachusetts, USA. 'Department of Pathology, \\ Brigham and Women's Hospital, Boston, Massachusetts, USA. ${ }^{3}$ Howard Hughes Medical Institute, Boston, Massachusetts, USA.
}

\begin{abstract}
Hereditary hemochromatosis is an iron-overload disorder resulting from mutations in proteins presumed to be involved in the maintenance of iron homeostasis. Mutations in hemojuvelin (HJV) cause severe, early-onset juvenile hemochromatosis. The normal function of HJV is unknown. Juvenile hemochromatosis patients have decreased urinary levels of hepcidin, a peptide hormone that binds to the cellular iron exporter ferroportin, causing its internalization and degradation. We have disrupted the murine $\mathrm{Hj} v$ gene and shown that $\mathrm{Hj}^{-/-}$mice have markedly increased iron deposition in liver, pancreas, and heart but decreased iron levels in tissue macrophages. Hepcidin mRNA expression was decreased in $\mathrm{Hjv}^{-/-}$mice. Accordingly, ferroportin expression detected by immunohistochemistry was markedly increased in both intestinal epithelial cells and macrophages. We propose that excess, unregulated ferroportin activity in these cell types leads to the increased intestinal iron absorption and plasma iron levels characteristic of the juvenile hemochromatosis phenotype.
\end{abstract}

\section{Introduction}

Hereditary hemochromatosis disorders result from mutations in genes coding for proteins that regulate iron homeostasis (reviewed in ref. 1). To date, pathological mutations have been discovered in 5 genes: HFE (encoding HFE), TFR2 (encoding transferrin receptor-2), SLC40A1 (encoding ferroportin), HAMP (encoding hepcidin), and $H J V$ (encoding hemojuvelin) (2-7). The autosomal-recessive diseases associated with mutations in HFE, TFR2, HAMP, and $H J V$ have similar clinical presentations. All are characterized by systemic iron overload with deposition of excess iron in parenchymal cells of the liver, heart, pancreas, and other endocrine tissues. Hemochromatosis disorders due to HFE and TFR2 mutations tend to be less severe, with later clinical onset, than those caused by HAMP and HJV mutations; the latter are characterized by accelerated iron loading, which, if untreated, may be lethal before the fourth decade of life.

While the normal roles of HFE, TFR2, and HJV have not yet been elucidated, 2 important clues suggest a unifying model for the pathogenesis of hemochromatosis. First, the 4 hemochromatosis disorders are all characterized by increased intestinal iron absorption and increased levels of serum iron. This suggests a defect that affects both absorptive enterocytes and tissue macrophages, which supply most of the serum iron primarily through recovery of the metal from senescent erythrocytes (1). Second, patients with all 4 disorders produce inappropriately low amounts of hepcidin, an iron-regulatory hormone (6-9). In normal individuals, hepcidin levels correlate with total body iron status - they are decreased in cases of iron deficiency and elevated in cases of iron overload (reviewed in ref. 1). In hemochromatosis patients, the deficit in hepcidin expression roughly correlates with the clinical severity of iron overload, which suggests that the normal hepcidin response is defective. Patients with mutations in HAMP and Hamp ${ }^{-1-}$ mice develop severe hemochromatosis associated with profound deficiency of hepcidin $(7,10,11)$. Con-

Nonstandard abbreviations used: HJV, hemojuvelin; RGM, repulsive guidance molecule; TFR2, transferrin receptor-2.

Conflict of interest: The authors have declared that no conflict of interest exists.

Citation for this article: J. Clin. Invest. 115:2187-2191 (2005).

doi:10.1172/JCI25049. versely, transgenic mice overexpressing hepcidin develop severe, prenatal iron-deficiency anemia and macrophage iron retention (12). These observations are all consistent with the hypothesis that hepcidin normally serves to inhibit intestinal iron absorption and macrophage iron release.

Recently, it was shown that hepcidin acts to downregulate cellsurface expression of ferroportin (13), a transmembrane iron transporter necessary for iron transfer out of intestinal epithelial cells and macrophages (14). In the absence of hepcidin, epithelial cells transfer more dietary iron across their basolateral membranes, which increases net absorption. Similarly, iron-recycling macrophages retain less storage iron and return more of it to the circulation. The consequence of both changes is hypersaturation of the serum iron carrier transferrin, which leads to a surfeit of non-transferrin-bound iron that is readily assimilated by parenchymal cells of the liver, heart, and endocrine tissues.

The role of HJV in iron homeostasis is obscure. Its closest homologs are repulsive guidance molecules (RGMs) RGM-A and RGM-B, membrane-tethered proteins important in the developing nervous system (15-19). Molecules in this family share structural features including an N-terminal signal peptide, an RGD motif, a von Willebrand type D domain, a GPI anchor motif, and a C-terminal transmembrane segment. While expression of RGM-A and RGM-B is restricted to the nervous system, $H J V$ mRNA is detectable in most tissues (20) and is expressed at high levels in skeletal muscle, cardiac muscle, and liver (6). More than 25 disease-causing mutations in HJV have been identified throughout the protein (21).

In this report, we describe the development of a mouse model of HJV hemochromatosis. We disrupted the murine Hjv gene through targeted deletion of most of the coding sequence. We found that $\mathrm{Hjv}^{-1-}$ mice rapidly accumulated iron in the liver, pancreas, and heart but had decreased iron content in the spleen. In contrast to previous findings in human patients, we detected no abnormalities in fertility and no obvious cardiac or endocrine abnormalities, which suggests that mice are more resistant to end-organ damage. Hepatic hepcidin expression was markedly decreased, and ferroportin protein was present at very high levels. These results support the hypothesis that juvenile hemochromatosis results from impaired hepcidin regulation and consequent overexpression of ferroportin. 

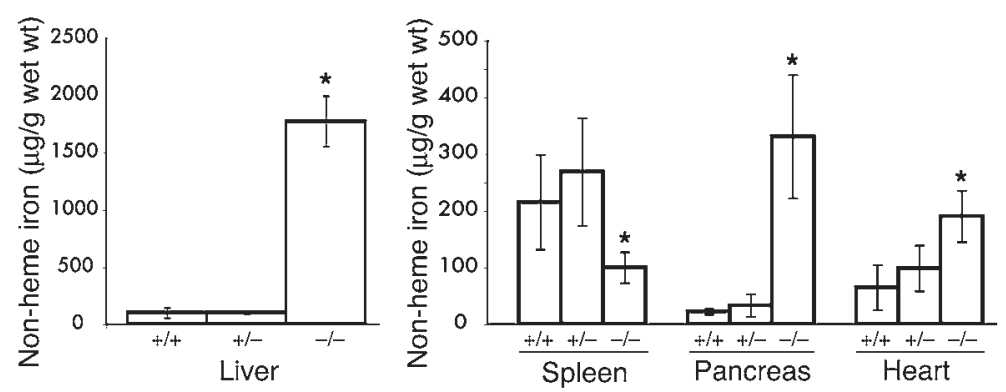

\section{Figure 1}

Elevated tissue iron content in $\mathrm{Hjv}^{-1-}$ mice. Non-heme tissue iron content $(\mu \mathrm{g} / \mathrm{g}$ wet $\mathrm{wt})$ was compared in wild-type $(+/+, n=6), \mathrm{HjV}^{+/-}(+/-, n=7)$, and $\mathrm{HjV}^{-/-}(-/-, n=4)$ mice at 6 weeks of age. ${ }^{*} P<0.05$ between $\mathrm{Hjv}^{-1-}$ and wild-type mice; Student's $t$ test.

\section{Results}

Targeted disruption of Hjv results in severe hemochromatosis. To disrupt the normal Hjv allele, we replaced exon 3 and the coding portion of exon 4 with a neomycin resistance cassette using homologous recombination in J1 ES cells (Supplemental Figure 1; supplemental material available online with this article; doi:10.1172/ JCI25049DS1). This knockout strategy deletes most of the sequence coding for the Hjv protein. We identified correctly targeted clones by Southern blot analysis and verified homologous recombination at both ends of the targeted region. We obtained chimeric animals, which were bred with 129S6/SvEvTac females to produce inbred $\mathrm{F}_{1}$ offspring carrying the modified $H j v$ allele. We confirmed germline transmission of the targeted allele by Southern blot and/or PCR analysis (data not shown).

We interbred $\mathrm{Hjv}^{+/-}$animals to produce $\mathrm{Hjv}^{-/-}$offspring, and approximately $25 \%$ of the offspring were $\mathrm{Hjv}^{-/-}$, which indicates that there was no significant prenatal lethality. The mutant mice were grossly indistinguishable from their littermates at birth. They developed normally and showed no evidence of neurological or muscular abnormalities. By 4 weeks of age, the livers of $\mathrm{Hjv}^{-/-}$mice were noticeably brown. We measured tissue iron content at 6 to 7 weeks of age and found that $\mathrm{Hjv}^{-/-}$mice had almost 20-fold more non-heme liver iron than did wild-type or $\mathrm{Hjv}^{+/-}$mice (Figure 1). Pancreatic iron accumulation was also markedly increased in $\mathrm{Hj}^{-/-}$mutants (Figure 1). Excess non-heme iron was present in the heart (Figure 1) as well as in the kidney and testis (data not shown). However, splenic non-heme iron content was decreased in $\mathrm{Hjv}^{-/}$mice compared with that in $\mathrm{Hjv}^{+/-}$and wild-type mice (Figure 1). Serum transferrin saturations were close to $100 \%$ in $\mathrm{Hj}^{-/-}$ mice (data not shown). Hemoglobin levels were indistinguishable between $\mathrm{Hjv}^{-/-}$and wild-type mice (data not shown).

We further examined the sites of iron accumulation by staining histological sections for iron (Figure 2). At 6 to 7 weeks of age, there was considerable, pericanalicular iron accumulation in liver parenchymal cells (hepatocytes), while the resident macrophages (Kupffer cells) were spared. In most animals, hepatocellular iron deposition appeared to be slightly more prominent in the periportal region (Figure 2, A and B, and data not shown). Iron accumulation was also observed in the distal tubules of the kidney and the

\section{Figure 2}

Histological examination of iron loading. Tissue iron was detected by staining with Perls Prussian blue (blue stain) or DAB-enhanced Perls stain (brown stain). (A) Wild-type liver. (B) $\mathrm{Hjv}^{-1-}$ liver. (C) Wild-type spleen. (D) $\mathrm{Hjv}^{-1-}$ spleen. (E and $\mathbf{G}$ ) Wild-type proximal duodenum. (F and $\mathbf{H}) \mathrm{HjV}^{-/}$proximal duodenum. Duodendal photomicrographs were taken approximately $0.5 \mathrm{~cm}$ from the pylorus. Original magnification, $\times 100(\mathbf{C}-\mathbf{F})$ and $\times 400(\mathbf{A}, \mathbf{B}, \mathbf{G}$, and $\mathbf{H})$. exocrine and endocrine pancreas, in a subset of cells in the anterior pituitary, and in arteriolar endothelial cells of the testis (data not shown). Compared with that in wild-type mice, iron staining was minimal in $\mathrm{Hjv}^{-/-}$splenic macrophages (Figure 2, C and D). Furthermore, while there was abundant iron in enterocytes of 6to 7-week-old wild-type mice, there was a marked decrease in the amount of stainable iron present in proximal duodenal enterocytes in $\mathrm{Hjv}^{-1-}$ mice (Figure 2, E-H).

Young, female $\mathrm{Hjv}^{-1-}$ mice were fertile and able to give birth to pups that were indistinguishable from normal mice. Six- to 7-weekold $\mathrm{Hjv}^{-/-}$male mice had robust, complete spermatogenesis and normal numbers of Leydig and Sertoli cells and were also fertile (data not shown). We conclude that systemic iron overload does not affect the reproductive potential of young adult $\mathrm{Hjv}^{-/-}$mice.

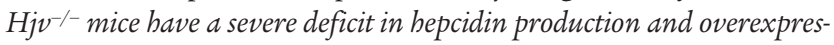
sion of ferroportin. It is not currently possible to measure serum

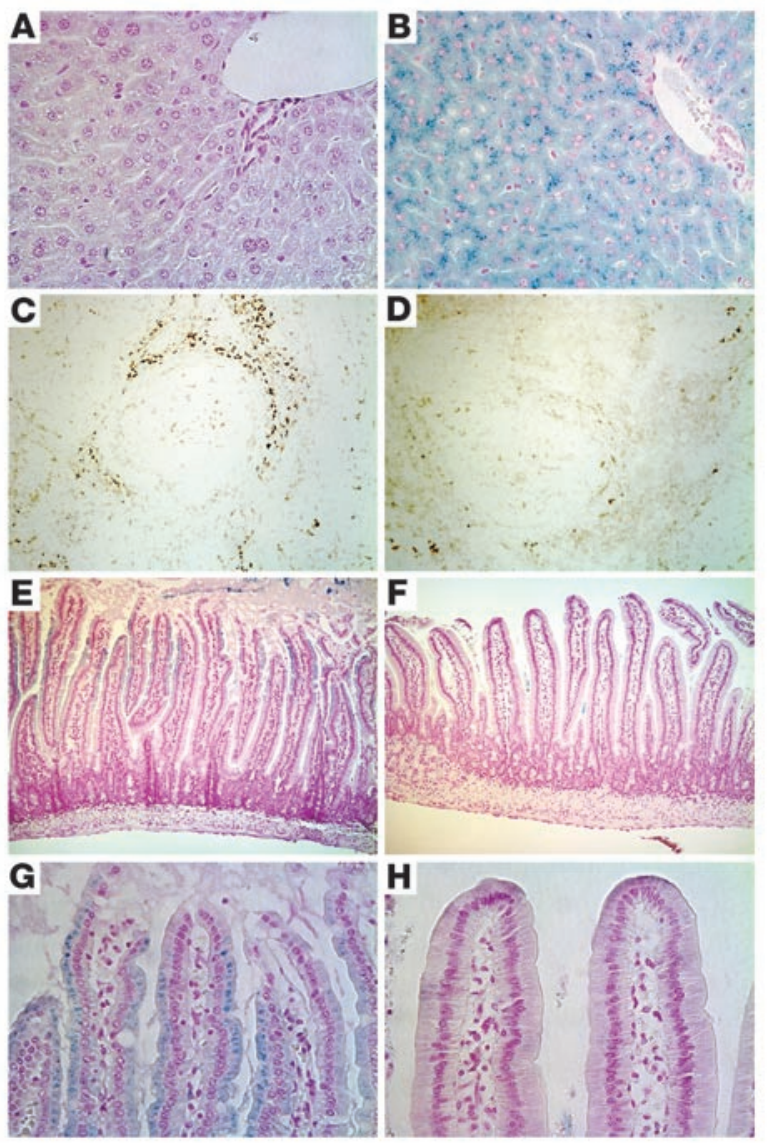




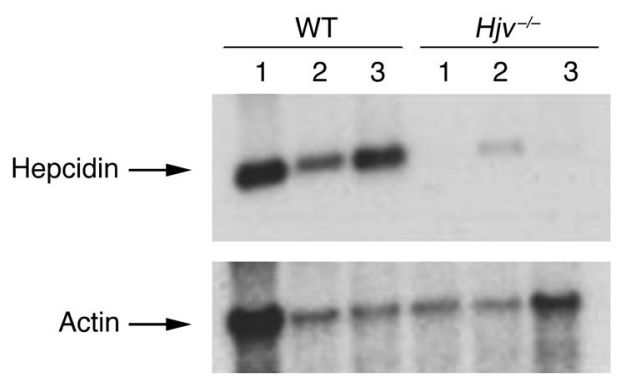

Figure 3

Expression of hepcidin mRNA. Hepatic mRNA was analyzed on a Northern blot probed for hepcidin and actin. Results are shown for 3 wild-type mice (left 3 lanes) and $3 \mathrm{Hjv}^{-1-}$ mice (right 3 lanes).

levels of hepcidin in mice. For that reason, we examined liver hepcidin mRNA expression by Northern blot analysis. We found that $\mathrm{Hj}^{-1-}$ mice had markedly decreased hepatic hepcidin mRNA expression as compared with that of control mice (Figure 3). Hepcidin mRNA was undetectable in some $\mathrm{Hj}^{-/-}$mice. When hepcidin mRNA was detected, the levels in $\mathrm{Hj}^{-/-}$animals averaged approximately 5.5-fold less than those observed in wild-type controls ( $P<0.005$, quantitation by phosphoimager analysis). The decrease in hepcidin mRNA levels in $\mathrm{Hjv}^{-/-}$mice occurred in spite of massive iron overload. Hepcidin mRNA levels in $\mathrm{Hjv}^{+/-}$mice were indistinguishable from those seen in wild-type controls (data not shown).

We examined ferroportin expression by immunohistochemistry, focusing on those tissues where ferroportin is known to be important (14). While immunohistochemistry is less quantitative than other techniques, it has the advantage of showing the cellular localization of the protein. Ferroportin is normally expressed at low levels in the absorptive enterocytes lining the intestinal villi (Figure 4, A and C). However, in $\mathrm{Hjv}^{-/-}$mice, we observed a massive increase in ferroportin protein expressed at the basolateral membrane (Figure 4, B and D). Similarly, ferroportin expression was markedly enhanced in tissue macrophages of the livers (Figure 4F) and spleens (Figure 4H) of $\mathrm{Hjv}^{-1}$ mice compared with that of wild-type controls (Figure 4, E and G).

\section{Discussion}

Targeted disruption of the murine Hjv gene resulted in a mouse phenotype similar to that of human patients with juvenile hemochromatosis. $\mathrm{Hjv}^{-/-}$mice rapidly accumulated excess iron in the liver, heart, and pancreas, which confirmed that HJV is the juvenile hemochromatosis gene and that loss of Hjv protein leads to iron overload. Interestingly, $\mathrm{Hjv}^{+/-}$mice were indistinguishable from wild-type mice in every study, which suggests that haploinsufficiency does not produce any clinical consequences. This is con-

\section{Figure 4}

Increased ferroportin expression in $\mathrm{HjV}^{-/-}$mice. Ferroportin expression was detected by immunohistochemistry. (A and $\mathbf{C}$ ) Wild-type proximal duodenum and (B and $\mathbf{D}$ ) $\mathrm{Hjv}^{-1-}$ proximal duodenum, approximately 0.5 $\mathrm{cm}$ from the pylorus, which is shown on the right in $\mathbf{A}$ and $\mathbf{B}$. Staining is most intense proximally in the wild-type animals but is expressed more intensely and along the distal two-thirds of the villus in the mutant animals. (E) Wild-type liver. (F) Hjv-l- liver. (G) Wild-type spleen. (H) $\mathrm{HjV}^{-1-}$ spleen. Note the intense Kupffer cell and macrophage staining in the $\mathrm{Hjv}^{-/-}$liver (F) and spleen (H). Original magnification, $\times 20$ (A and B), $\times 100$ (C, D, G, and H), and $\times 400$ (E and F). sistent with the fact that parents of juvenile hemochromatosis patients have never been reported to be affected but is notably different from previous observations in $\mathrm{Hfe}^{+/-}$mice, in which a mild heterozygote phenotype has been detected (22).

By 6 to 7 weeks of age, $\mathrm{Hjv}^{-/-}$mice had accumulated significantly more iron than 12-week-old $\mathrm{Hfe}^{-/-}$mice of the same genetic background (23), which is consistent with the difference in severity between juvenile hemochromatosis and HFE hemochromatosis in human patients. However, similar to other mouse models of iron overload (refs. 23-25, and our unpublished observations), $\mathrm{Hj}^{-/-}$ mice did not develop significant hepatic fibrosis, frank cardiomyopathy, overt diabetes, infertility, or other end-organ dysfunction. Mice appeared to be protected, by some unknown mechanism, from toxic effects of iron overload.

Although HJV is expressed at high levels in cardiac and skeletal myocytes, $\mathrm{Hjv}^{-/-}$mice, similar to juvenile hemochromatosis patients, had no grossly apparent abnormalities aside from iron overload, which suggests that Hjv plays no essential role in muscle development or function within the first 2 months of life. Based on this phenotype, we conclude that the critical role of HJV is to control iron homeostasis. Our data suggest that it acts by potentiating hepcidin expression. Hepcidin is primarily produced in the liver, but smaller amounts are made in the heart and pancreas (26), which is similar to the pattern of HJV expression (refs. 6, 20, and our unpublished observations). It is possible that HJV controls hepcidin expression at several of those sites. Intriguingly, HJV is expressed at the primary sites of iron loading in hemochromatosis,
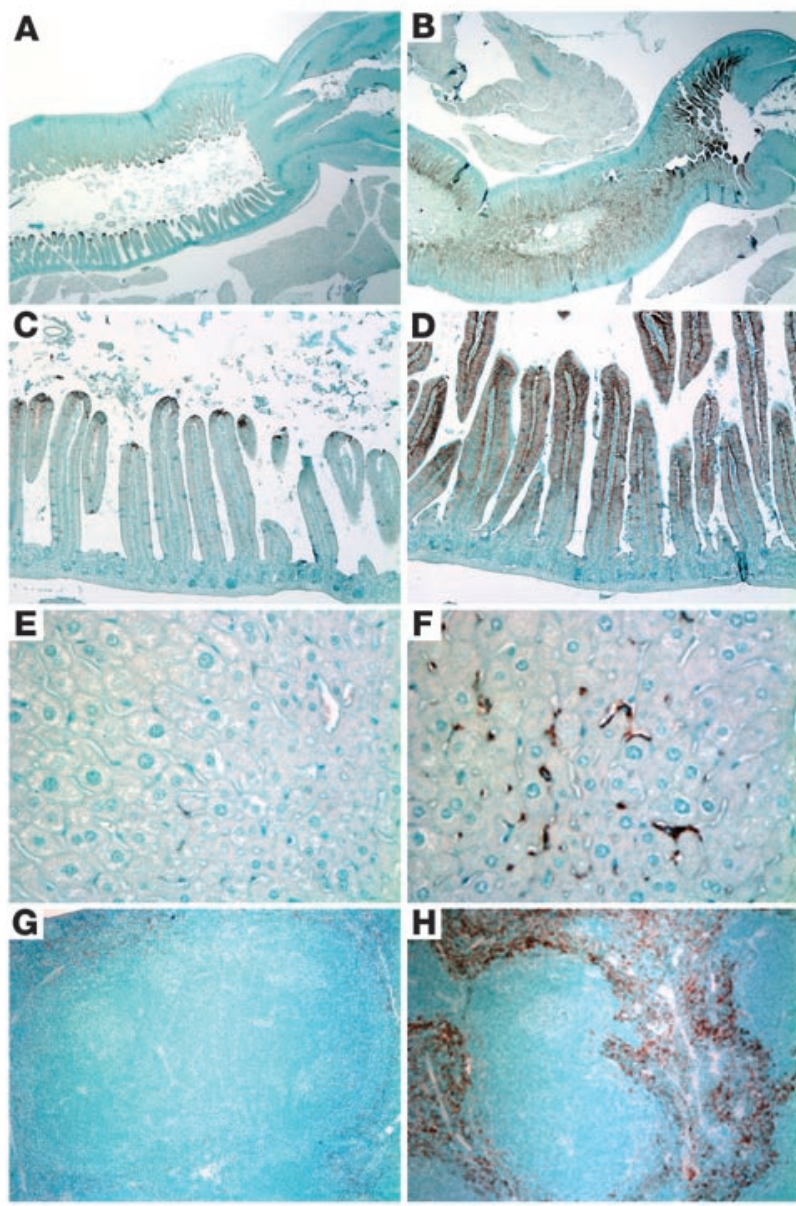
which suggests that it could have a second, cell-autonomous function in either sensing or directly modulating cellular iron uptake.

In the absence of appropriate hepcidin expression, we would expect impaired regulation of the iron exporter, ferroportin. Accordingly, we observed markedly elevated levels of ferroportin in 2 critical sites: the absorptive intestinal epithelium and tissue macrophages. The iron content of both enterocytes and macrophages was decreased compared with that of wild-type animals. Thus, increased intestinal ferroportin expression could account for accelerated iron absorption and increased body iron burden by maximizing the amount of enterocyte iron transferred to serum. Similarly, increased macrophage ferroportin expression could potentiate macrophage iron release, resulting in decreased macrophage storage iron and increased serum iron.

Our study also has implications for autosomal-dominant iron overload associated with mutations in the gene encoding ferroportin. There appear to be 2 classes of patients with "ferroportin disease" (27). One group has increased macrophage iron stores with no apparent increase in total body iron, which suggests a partial loss of ferroportin function $(4,28)$. Their phenotype is similar to that observed in mice lacking 1 ferroportin allele (14). However, other patients with a different constellation of ferroportin missense mutations present with symptoms that are more similar to those of the other hemochromatosis disorders $(29,30)$. These patients may have increased ferroportin activity similar to that of $\mathrm{Hj}^{-/-}$mice. This might result either from increased ferroportin transporter activity or from impaired binding of hepcidin.

In summary, our findings define a mechanism for iron overload in juvenile hemochromatosis due to mutations in HJV. Together with results of other studies showing decreased hepcidin expression in other hemochromatosis disorders, they suggest that increased body iron burden results from overactivity of ferroportin due to impaired regulation by hepcidin. We propose that increased ferroportin expression is the direct cause of increased intestinal iron absorption and increased serum iron, which leads to rapid deposition in target organs.

\section{Methods}

Targeted disruption of the murine Hjv gene. We isolated Hjv genomic clones from a strain 129/SvJ mouse library (Stratagene). To construct the targeting vector to delete exon 3 and the entire coding region of exon 4 of $\mathrm{Hjv}$, we subcloned fragments of $3.8 \mathrm{~kb}$ (upstream of the deleted region) and $2.9 \mathrm{~kb}$ (downstream) into a targeting vector containing a neomycin-resistance cassette flanked by loxP sites (Supplemental Figure 1). Linearized vector was electroporated into $129 \mathrm{~J} 1 \mathrm{ES}$ cells. Correct homologous recombination was confirmed by Southern blot or PCR analysis of flanking sequences from both ends of the targeted region. ES cell clones with normal karyotypes were injected into C57BL/ 6 blastocysts. Transmission of the targeted alleles was confirmed by Southern blot analysis. The animals were maintained on an inbred 129S6/SvEvTac background. Subsequent genotyping was carried out by extracting DNA from snipped tail samples (PureGene kit; Gentra Systems) and subjecting it to Southern blot and/or PCR analysis.

Animal care. All mice were born and housed in the barrier facility at Children's Hospital Boston. We weaned pups at 28 days and maintained them on Prolab RMH 3000 LabDiet (PMI Nutrition International), which has 380 parts per million of iron. All mouse procedures were approved by the Institutional Animal Care and Use Committee at Children's Hospital Boston.

Tissue iron staining and quantitative tissue iron measurements. Tissues were harvested from 6- to 7-week-old mice that were not fasted prior to sacrifice. Intestine, liver, and spleen samples were fixed in $10 \%$ buffered formalin and embedded in paraffin. Deparaffinized tissue sections were stained with the Perls Prussian blue stain for non-heme iron in the Children's Hospital Boston Pathology Laboratory. A subset of tissue sections were stained with a diaminobenzidine-enhanced Perls iron stain (31). Deparaffinized tissue sections were incubated for 30 minutes in $1 \%$ potassium ferrocyanide in $0.12 \mathrm{~N} \mathrm{HCl}$. Endogenous peroxidase activity was quenched for 20 minutes at room temperature in $0.3 \% \mathrm{H}_{2} \mathrm{O}_{2}$ in methanol. Sections were rinsed in PBS and incubated for 6 minutes in $\mathrm{DAB} / \mathrm{H}_{2} \mathrm{O}_{2}$ (DAB-Plus Substrate Kit; Zymed Laboratories Inc.). Quantitative measurement of non-heme iron was performed as described previously (22). Results are reported as micrograms of iron per gram wet weight of tissue.

RNA preparation and Northern blot analysis. We harvested liver tissue from 6- to 7-week-old mice at sacrifice and immediately stored it at $-80^{\circ} \mathrm{C}$ until it was immersed in RNAlater solution (Ambion Inc.). We then transferred the tissue from RNAlater to Trizol (Invitrogen Corp.) and homogenized it 3 times for 20 seconds each using a Polytron PT2100 apparatus (Novodirect $\mathrm{GmbH}$ ). We extracted RNA according to the manufacturer's instructions.

We fractionated $10 \mu \mathrm{g}$ of total RNA from individual $\mathrm{Hjv}^{-/-}$and wild-type mice on a $1 \%$ formaldehyde agarose gel and blotted it onto a nylon membrane (Hybond-N+; Amersham Biosciences). We hybridized the membrane with ${ }^{32} \mathrm{P}$-labeled probes specific for hepcidin and $\beta$-actin mRNAs. We quantified the signals on a fluoroimager (Molecular Dynamics).

Immunohistochemistry. Immunohistochemical staining of mouse tissues with an anti-ferroportin antibody (32) was carried out as described previously (14). Previous studies comparing immunoreactivity with this antibody and preimmune controls validated the specificity of the antibody (data not shown).

\section{Acknowledgments}

N.C. Andrews is an investigator of the Howard Hughes Medical Institute. M.D. Fleming is supported in part by NIH grants R01 DK 062474 and R01 DK 066373. F.W. Huang is an Albert J. Ryan Foundation Fellow at Harvard Medical School. We are grateful to Xenon Pharmaceuticals Inc. for providing data prior to publication. We thank Dean Campagna for technical assistance in subcloning and Angel Custodio for assistance with retro-orbital phlebotomy. We thank Paul Schmidt, Cindy Roy, and Adriana Donovan for helpful discussions. ES cell manipulations and blastocyst injections were carried out by the Mental Retardation Research Center Gene Manipulation Facility at Children's Hospital, funded in part by NIH grant P30 HD18655.

Received for publication March 16, 2005, and accepted in revised form May 10, 2005.

Address correspondence to: Nancy Andrews, Children's Hospital, Karp Family Research Laboratories, RM 8-125, 300 Longwood Avenue, Boston, Massachusetts 02115-5737, USA. Phone: (617) 919-2116; Fax: (617) 432-3639; E-mail: nancy_andrews@hms. harvard.edu.

F.W. Huang designed and executed all experiments described in this report and contributed to writing the manuscript.

J.L. Pinkus and G.S. Pinkus carried out the immunohistochemistry experiments.

M.D. Fleming assisted in analysis of pathology.

N.C. Andrews and M.D. Fleming guided the overall development of the research plan and contributed to the writing of the manuscript. 
1. Hentze, M.W., Muckenthaler, M.U., and Andrews, N.C. 2004. Balancing acts: molecular control of mammalian iron metabolism [review]. Cell. 117:285-297.

2. Camaschella, C., et al. 2000. The gene TFR2 is mutated in a new type of haemochromatosis mapping to 7q22. Nat. Genet. 25:14-15.

3. Feder, J.N., et al. 1996. A novel MHC class I-like gene is mutated in patients with hereditary haemochromatosis. Nat. Genet. 13:399-408.

4. Montosi, G., et al. 2001. Autosomal-dominant hemochromatosis is associated with a mutation in the ferroportin (SLC11A3) gene. J. Clin. Invest. 108:619-623. doi:10.1172/JCI200113468.

5. Njajou, O.T., et al. 2001. A mutation in SLC11A3 is associated with autosomal dominant hemochromatosis. Nat. Genet. 28:213-214.

6. Papanikolaou, G., et al. 2004. Mutations in HFE2 cause iron overload in chromosome 1q-linked juvenile hemochromatosis. Nat. Genet. 36:77-82.

7. Roetto, A., et al. 2003. Mutant antimicrobial peptide hepcidin is associated with severe juvenile hemochromatosis. Nat. Genet. 33:21-22.

8. Bridle, K.R., et al. 2003. Disrupted hepcidin regulation in HFE-associated haemochromatosis and the liver as a regulator of body iron homoeostasis. Lancet. 361:669-673.

9. Nemeth, E., Roetto, A., Garozzo, G., Ganz, T., and Camaschella, C. 2005. Hepcidin is decreased in TFR2 hemochromatosis. Blood. 105:1803-1806.

10. Nicolas, G., et al. 2001. Lack of hepcidin gene expression and severe tissue iron overload in upstream stimulatory factor 2 (USF2) knockout mice. Proc. Natl. Acad. Sci. U. S. A. 98:8780-8785.

11. Viatte, L., et al. 2005. Deregulation of proteins involved in iron metabolism in hepcidin-deficient mice. Blood. doi:10.1182/blood-2004-12-4608.

12. Nicolas, G., et al. 2002. Severe iron deficiency ane- mia in transgenic mice expressing liver hepcidin. Proc. Natl. Acad. Sci. U. S. A. 99:4596-4601.

13. Nemeth, E., et al. 2004. Hepcidin regulates cellular iron efflux by binding to ferroportin and inducing its internalization. Science. 306:2090-2093.

14. Donovan, A., et al. 2005. The iron exporter ferroportin (Slc40a1) is essential for iron homeostasis. Cell Metabolism. 1:191-200.

15. Brinks, H., et al. 2004. The repulsive guidance molecule RGMa is involved in the formation of afferent connections in the dentate gyrus. J. Neurosci. 24:3862-3869.

16. Matsunaga, E., et al. 2004. RGM and its receptor neogenin regulate neuronal survival. Nat. Cell Biol. 6:749-755.

17. Monnier, P.P., et al. 2002. RGM is a repulsive guidance molecule for retinal axons. Nature. 419:392-395.

18. Niederkofler, V., Salie, R., Sigrist, M., and Arber, S. 2004. Repulsive guidance molecule (RGM) gene function is required for neural tube closure but not retinal topography in the mouse visual system. J. Neurosci. 24:808-818.

19. Samad, T.A., et al. 2004. DRAGON: a member of the repulsive guidance molecule-related family of neuronal- and muscle-expressed membrane proteins is regulated by DRG11 and has neuronal adhesive properties. J. Neurosci. 24:2027-2036.

20. Rodriguez Martinez, A., Niemela, O., and Parkkila, S. 2004. Hepatic and extrahepatic expression of the new iron regulatory protein hemojuvelin. Haematologica. 89:1441-1445.

21. Lanzara, C., et al. 2004. Spectrum of hemojuvelin gene mutations in 1q-linked juvenile hemochromatosis. Blood. 103:4317-4321.

22. Levy, J.E., Montross, L.K., Cohen, D.E., Fleming, M.D., and Andrews, N.C. 1999. The C282Y mutation causing hereditary hemochromatosis does not produce a null allele. Blood. 94:9-11.

23. Levy, J.E., Montross, L.K., and Andrews, N.C. 2000. Genes that modify the hemochromatosis phenotype in mice. J. Clin. Invest. 105:1209-1216.

24. Levy, J.E., Jin, O., Fujiwara, Y., Kuo, F., and Andrews, N.C. 1999. Transferrin receptor is necessary for development of erythrocytes and the nervous system. Nat. Genet. 21:396-399.

25. Trenor, C.C., 3rd, Campagna, D.R., Sellers, V.M., Andrews, N.C., and Fleming, M.D. 2000. The molecular defect in hypotransferrinemic mice. Blood. 96:1113-1118.

26. Ilyin, G., et al. 2003. Comparative analysis of mouse hepcidin 1 and 2 genes: evidence for different patterns of expression and co-inducibility during iron overload. FEBS Lett. 542:22-26.

27. Schimanski, L.M., et al. 2005. In vitro functional analysis of human ferroportin (FPN) and hemochromatosis-associated FPN mutations. Blood. 105:4096-5102.

28. Devalia, V., et al. 2002. Autosomal dominant reticuloendothelial iron overload associated with a 3-base pair deletion in the ferroportin 1 gene (SLC11A3). Blood. 100:695-697.

29. Arden, K.E., et al. 2003. A novel mutation in ferroportin 1 is associated with haemochromatosis in a Solomon Islands patient. Gut. 52:1215-1217.

30. Njajou, O.T., et al. 2002. Dominant hemochromatosis due to $\mathrm{N} 144 \mathrm{H}$ mutation of SLC11A3: clinical and biological characteristics. Blood Cells Mol. Dis. 29:439-443.

31. Dragatsis, I., Efstratiadis, A., and Zeitlin, S. 1998. Mouse mutant embryos lacking huntingtin are rescued from lethality by wild-type extraembryonic tissues. Development. 125:1529-1539.

32. Donovan, A., et al. 2000. Positional cloning of zebrafish ferroportin 1 identifies a conserved vertebrate iron exporter. Nature. 403:776-781. 